as appears probable, this is typical of Atlantic surface water at midwinter, the 'Channel' (setosa)1 water, characterized by the presence of the chætognath, Sagitta setosa, which has occupied the English Channel in the neighbourhood of Plymouth since 1930-31 and has similar maximum phosphate content $\left(0.42-0.48 \mathrm{mgm}\right.$.-atom $\left./ \mathrm{m}^{3} \mathrm{P}\right)$, may well have been derived from it. We should, however, have to look elsewhere for the origin of the higher phosphate content $(0 \cdot 6-0.7$ mgm.-atom) of the 'elegans' or 'western' water ${ }^{1}$ of the Celtic Sea, which in the nineteen twenties also filled the western end of the English Channel. The most probable source is upwelling of deeper ocean water from the continental slope. Such upwelling may be attributed to off-shore winds, which would need to be from the north and east. South-westerlies are the prevailing winds of the area both in direction and strength, and the winds from the northern and eastern sectors would, in general, seem not to blow hard enough or long enough to produce upwelling on the scale required. Periods of prolonged fresh easterly winds such as February 1947 are rather exceptional.

There is another possible mechanism by which nutrients could be brought from the deeper waters on to the continental shelf. The existence of internal or boundary waves in the ocean with an amplitude up to 100 metres is now well established. If such waves should impinge at right angles to a vertical cliff, no doubt they would be totally reflected. The continental slope, though steep, is far from vertical, so that the energy of an impinging wave must be dissipated in some other way. Most probably such a wave would run up the slope much as a surface wave runs up a shelving beach. In this way nutrientricher water at mid-depths could be brought higher on to the shelf, where normal vertical mixing due to wind-waves and surface winter cooling would complete the process of bringing the richer water to the surface.

No evidence has yet been adduced to show whether or not submarine valleys or canyons such as those found on the New England coast are present along the continental slope west of the English Channel. If such exist, they would concentrate the energy of the internal wave into a contracting cross-section and greatly amplify the power of an internal wave to project deeper water into the surface layers. For a full understanding of the fertility of the sea in this area, a synoptic survey of the internal waves in the Atlantic abreast of the slope and a knowledge of the topography $\mathrm{cf}$ the slope itself is therefore called for.

Marine Biological Laboratory, L. H. N. COOPER

Plymouth.

March 5.

${ }^{1}$ Russell, F. S., Cons. Perm. Int. Expl. Mer, Rapp. Proc.-verb., 100, 7 (1936); J. Mar. Biol. A880c., 20, 309 (1935).

\section{Relative Directions of the Electric and Mag- netic Vectors in Electromagnetic Waves in vacuo}

ARE the electric vector $(\vec{E})$ and the magnetic vector $(\vec{B})$ in electromagnetic waves in vacuo always mutually perpendicular, or are they not always so ?

I put this question to a number of distinguished physicists, and all of them, without exception, answered definitely in the affirmative at first-and then agreed with me that the correct answer should be in the negative.

It follows from the Maxwell equations

$$
\vec{E}=c \text { curl } \vec{B} \quad \overrightarrow{\vec{B}}=-c \operatorname{curl} \vec{E}
$$

that the time derivative vector of $\vec{E}$-not the vector itself-is perpendicular to $\vec{B}$ and, correspondingly, the time derivative vector of $\vec{B}$-not the vector itself -is perpendicular to $\vec{E}$. But the directions of the vector and of its time derivative always coincide only in the case of a non-rotating vector. This is, however, as a rule, not so in the case of a rotating vector (take, for example, the acceleration and the velocity in curvilinear motion).

Thus, in plane polarized plane electromagnetic waves, where the vectors $\vec{E}$ and $\vec{B}$ do not rotate, they are mutually perpendicular; but in rotating electromagnetic waves ${ }^{1}$ and in electromagnetic whirls ${ }^{2}$, where these vectors rotate, they are, generally speaking, not mutually perpendicular. Only in some exceptional cases are $\vec{E}$ and $\vec{B}$ perpendicular, in spite of rotation. This happens, for example, in circularly polarized plane waves, where both vectors are coplanar and rotate with equal angular velocity always at right angles to each other; and in spherical or in cylindrical waves, where one of the vectors rotates in an axial plane, while the other vector remains in the same position in an angular plane $e^{1,2,3}$.

Apparently this point is usually overlooked, because until quite recently very little attention was given to the study of electromagnetic waves with rotating vectors other than plane and spherical waves.

Royal Institution,

N. S. JAPOLSKY

21 Albemarle Street, London, W.1.

Feb. 15.

${ }^{1}$ Phil. Mag., 19, 934 (1935).

${ }^{2}$ Phil. Mag., 20, 417 (1935).

${ }^{3}$ Nature, 137, 582 and 1031 (1936).

\section{Origin of Solar 'Static'}

Recentuy, Martyn ${ }^{1}$ wrote: "It is natural first to seek an explanation [of the origin of solar static] in terms of the kind of electrical discharge which causes the familiar terrestrial radio noise, namely, lightning flashes; but it is difficult, if not impossible, to see how such discharges could be produced in the always highly ionized solar envelope". The explanation to which Martyn raises objection is, in fact, one which I have myself put forward recently ${ }^{2}$. His objection, however, does not arise on the general theory which I have advanced elsewhere ${ }^{3}$. Far from preventing the existence of electric fields at the sun's surface, the thermal conditions there are themselves the result of electrical discharges. These discharges occur in the radial electric field set up by the emission of highly charged atoms from nuclear reactions in the sun's interior.

Chas. E. R. Bruce

British Electrical and Allied Industries

Research Association,

5 Wadsworth Road,

Greenford, Middx. Feb. 26.

${ }^{1}$ Martyn, D. F., Nature, 159, 27 (1947).

${ }^{2}$ Bruce, C. E. R., Observatory, 68, 263 (1946).

3 Bruce, C. E. R. "A New Approach in Astrophysics and Cosmogony" (London, 1944). 